\title{
The Role of the Left Head of Caudate in Suppressing Irrelevant Words
}

\author{
Nilufa Ali, David W. Green, Ferath Kherif, Joseph T. Devlin, \\ and Cathy J. Price
}

\begin{abstract}
Suppressing irrelevant words is essential to successful speech production and is expected to involve general control mechanisms that reduce interference from task-unrelated processing. To investigate the neural mechanisms that suppress visual word interference, we used fMRI and a Stroop task, using a block design with an event-related analysis. Participants indicated with a finger press whether a visual stimulus was colored pink or blue. The stimulus was either the written word "BLUE," the written word "PINK," or a string of four Xs, with word interference introduced when the meaning of the word and its color were "incongruent" (e.g., BLUE in pink hue) relative to congruent (e.g., BLUE in blue) or neutral (e.g., XXXX in pink). The participants also made color decisions in the presence of spatial
\end{abstract}

\section{INTRODUCTION}

To say what we want to say, our intended words must overcome interference from competing words with similar sounds or meanings. Control mechanisms are therefore required to suppress interference from unintended words while enhancing activation for the intended words. Previous functional imaging experiments have investigated the impact of word interference on brain activation by manipulating the level of word interference during picture processing (Hocking, McMahon, \& de Zubicaray, 2008; Abel et al., 2009; Rahman \& Melinger, 2008; de Zubicaray, McMahon, Eastburn, \& Pringle, 2006), speech comprehension (Hoenig \& Scheef, 2009; Mason \& Just, 2007; Zempleni, Renken, Hoeks, Hoogduin, \& Stowe, 2007; Rodd, Davis, \& Johnsrude, 2005), or color naming (Haupt, Axmacher, Cohen, Elger, \& Fell, 2009; Melcher \& Gruber, 2009; Liu, Bai, \& Zhang, 2008; Polk, Drake, Jonides, Smith, \& Smith, 2008; Roberts \& Hall, 2008; Egner, Delano, \& Hirsch, 2007; Langenecker, Nielson, \& Rao, 2004; Liu, Banich, Jacobson, \& Tanabe, 2004; Peterson et al., 1999). The results typically highlight the role of dorsolateral prefrontal cortex and/or the anterior cingulate but many other regions have been implicated depending on the task and context (Spalek \& Thompson-Schill, 2008) and the exact

University College London, London, UK interference rather than word interference (i.e., the Simon task). By blocking incongruent, congruent, and neutral trials, we identified activation related to the mechanisms that suppress interference as that which was greater at the end relative to the start of incongruency. This highlighted the role of the left head of caudate in the control of word interference but not spatial interference. The response in the left head of caudate contrasted to bilateral inferior frontal activation that was greater at the start than at the end of incongruency, and to the dorsal anterior cingulate gyrus which responded to a change in the motor response. Our study therefore provides novel insights into the role of the left head of caudate in the mechanisms that suppress word interference.

nature of the question being asked. In this study, our aim was to investigate the neural mechanisms that suppress word interference. In part, our interest was driven by the relevance of this question to the understanding of bilingualism because speaking in a nondominant language requires suppression of interference from the dominant (usually native) language (e.g., Rodriguez-Fornells et al., 2005; Rodriguez-Fornells, Rotte, Heinze, Nösselt, \& Münte, 2002). As a first step, however, the current study focuses on the mechanisms that suppress visual word interference, within language, in monolingual speakers.

Our experimental design needed to dissociate activation related to the mechanisms that suppress interference from activation related to the presence of uncontrolled interference (= conflict). This requirement was met by using fMRI and a Stroop task with a block design and an event-related analysis. As in the standard Stroop task (MacLeod, 1991; Stroop, 1935), we instructed participants to respond to the print color of a visual stimulus that was either a color word (BLUE or PINK) or a string of four Xs (XXXX). In this paradigm, word interference is introduced when the word indicates a color that is incongruent from the hue of the physical stimulus (e.g., the word PINK written in blue). Although the participant is only instructed to respond to the physical hue, word interference arises because reading is an overly learned skill that proceeds to the level of covert speech production even when this is not explicitly required 
by the task (Price, Wise, \& Frackowiak, 1996; Van Orden, 1987). Activation related to word interference in the Stroop color naming task was therefore expected to be higher during these incongruent trials relative to congruent trials, where the word corresponds to the physical hue (e.g., the word PINK written in pink or the word BLUE written in blue) or neutral trials where the stimulus is unrelated to the physical hue (XXXX presented in blue or pink). Like previous fMRI studies of the Stroop task, our subjects indicated the color of the stimulus using a manual response (e.g., Egner \& Hirsch, 2005a; van Veen \& Carter, 2005; Liu et al., 2004; Mead et al., 2002; Peterson et al., 2002).

Successful performance on the Stroop task in the context of word interference/conflict, during the incongruent trials, will necessitate increased involvement from mechanisms that suppress interference. To dissociate processing related to word conflict from processing related to the mechanisms that suppress it, we examined the effect of an incongruent trial on a subsequent incongruent trial (e.g., Wuhr \& Ansorge, 2005) because conflict and control are expected to follow different time courses. According to the conflict adaptation theory (e.g., Egner \& Hirsch, 2005a; Botvinick, Braver, Barch, Carter, \& Cohen,
2001), control processes are triggered by the detection of conflict and these control processes then modulate the source of conflict. Hence, when several incongruent trials are presented one after another, activation related to conflict is expected to be higher on the first trial than on subsequent trials, whereas activation related to the control of interference is expected to be lower on the first trial than on subsequent trials (Durston et al., 2003; see Figure 1, for an illustration of changes in activation related to control that are either step-like or gradual). In terms of task performance, this was expected to be worse on the first incongruent trial (more errors/slower RTs) but to improve on subsequent incongruent trials when control mechanisms take effect to suppress word interference (e.g., Wuhr \& Ansorge, 2005; Kerns et al., 2004).

Our experimental design therefore involved short blocks of stimuli that were incongruent, congruent, or neutral. The block design contrasts with the majority of fMRI studies of the Stroop task, that have deliberately randomized different trial types to prevent participants guessing the next trial (Sumner et al., 2007; Egner \& Hirsch, 2005a; Nieuwenhuis \& Yeung, 2005; Kastner \& Ungerleider, 2000; Desimone \& Duncan, 1995; Cohen,
Figure 1. Predictions for activation related to conflict and its control during incongruent trials. Top shows expectation that activation related to conflict and control will (A) increase at the start of a run of incongruent trials and (B) decrease at the end of a run of incongruent trials. $\mathrm{C}=$ run of congruent trials. $\mathrm{I}=$ run of incongruent trials. Lower two rows show the differing expectations for conflict and control. The expectation is that conflict will be higher at the start of a run of incongruent trials than the end of a run of congruent trials, that is, A will be greater than $\mathrm{B}$. In contrast, control is expected to be higher at the end of a run than the beginning of the run, that is, $\mathrm{B}$ will be greater than $\mathrm{A}$. The shape of these effects across an incongruent run could either be gradual (middle row) or primarily occur on the second run of a trial and then plateau for the rest of the run (lower row). The changing effect of control during a congruent or neutral run could also be gradual or stepwise, however, our experimental design and analysis do not allow us to determine how activation related to control changed over trials of the same type.

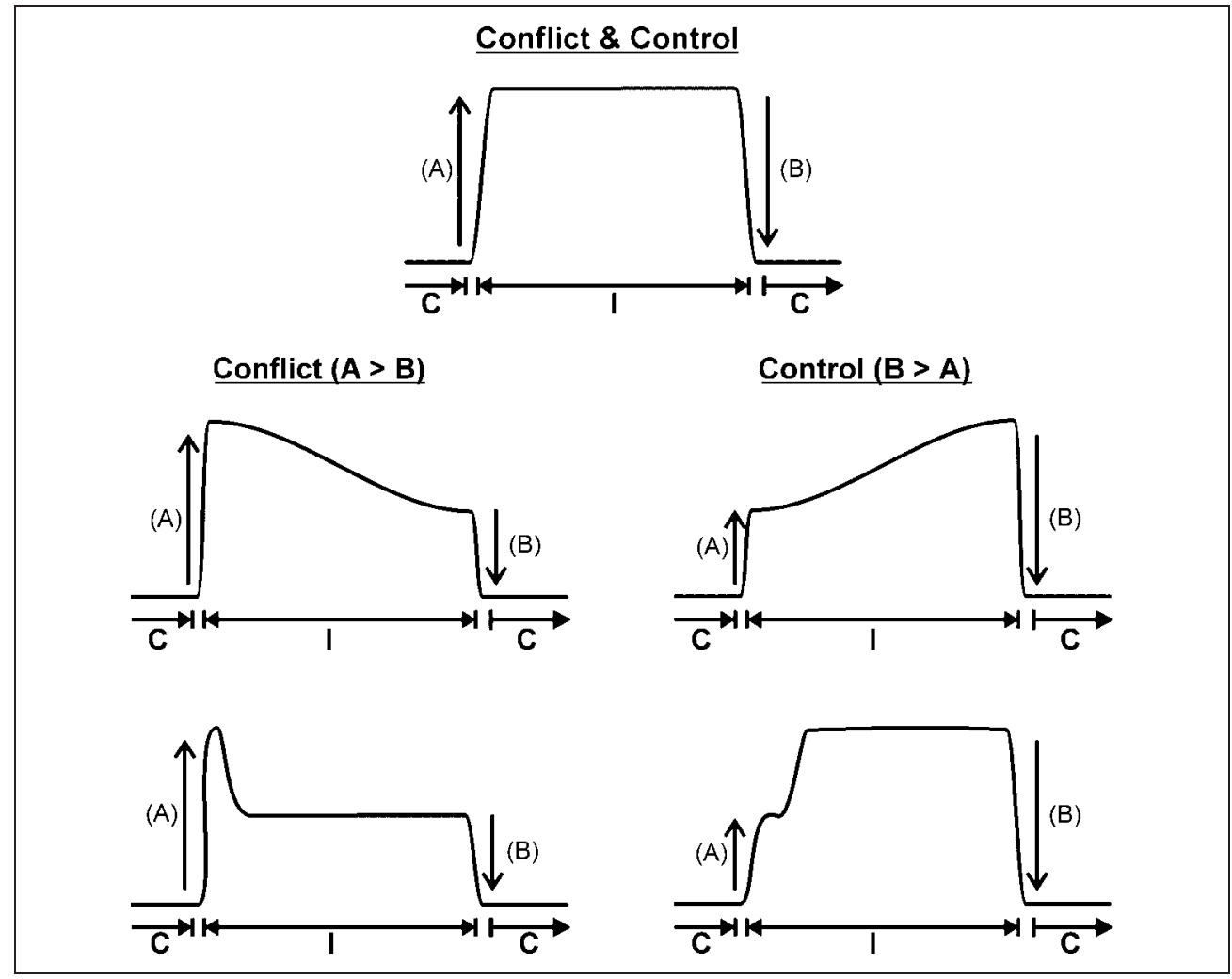


Dunbar, \& McClelland, 1990), although other fMRI studies have also investigated the effect of prior context on incongruent responses (Liu et al., 2008; Roberts \& Hall, 2008; Botvinick et al., 2001). Our blocked design allowed us to compare activation at the start and the end of a run of incongruent trials and thereby dissociate activation related to conflict and its control. We also dissociated activation related to a change in the required motor response (i.e., left finger vs. right finger responses to indicate stimulus color) by ensuring that motor response changes always occurred within a block and never coincided with a change in interference. By dissociating effects related to a change in motor response from effects related to word interference, we increased sensitivity because, if these effects were not modeled independently, they could both be lost to error variance if they were located in close proximity.

Critically, the control of interference in the Stroop task involves processes other than word inhibition (Melcher \& Gruber, 2009). For example, control mechanisms might amplify task-relevant processing (the color of the stimulus) and its mapping to the response (see Polk et al., 2008; Egner \& Hirsch, 2005a; Kastner \& Ungerleider, 2000; Desimone \& Duncan, 1995; Cohen et al., 1990) or the motor activation associated with it (see Sumner et al., 2007) To characterize and dissociate control processes that were not related to word inhibition, we compared our findings from the Stroop task with those from the Simon task ( $\mathrm{Lu}$ \& Proctor, 1995; Simon, 1969). In the Simon task, the instructions were identical to those used in the Stroop task (i.e., respond with one finger if the stimulus is colored blue and another finger if the stimulus is colored pink). However, the type of interference is not the same because the stimuli were not words but colored rectangles that varied in the relationship between their spatial position and the required keypress. In this context, incongruent trials were those where a blue stimulus, requiring a left key response, was presented right of fixation; or a pink stimulus, requiring a right key response, was presented left of fixation. Conflict is therefore at the level of the side of response. As in the Stroop task, we included congruent and neutral trials as well as incongruent trials with the expectation that interference would be higher during the incongruent trials when the response is incompatible with the physical location of the stimulus.

Although both the Simon and Stroop tasks involve the same "relevant" information (i.e., color processing), the type of interference and the mechanisms that control it were expected to differ (see Egner et al., 2007). By comparing the effects of control in the Simon and Stroop tasks, we dissociated common effects of control from those that were specific to the Stroop task and possibly related to word inhibition. Only three neuroimaging studies, to date, have directly compared Stroop and Simon effects (Egner et al., 2007; Fan, Flombaum, McCandliss, Thomas, \& Posner, 2003; Peterson et al., 2002) and, as expected, their results highlighted both commonalities and differences between tasks. However, in contrast to our study, none focused on the control of word interference or dissociated the effects of conflict, its control, and response change.

\section{Predictions}

As there were many novel features in our experimental design, we searched the whole brain in an unbiased manner for effects that were consistent with a role in the mechanisms that suppress word interference. Nevertheless, we were interested in the pattern of activation in two cortical regions and one subcortical region. Dorsolateral prefrontal cortex and the anterior cingulate are regions that have been most frequently associated with incongruent trials in previous functional imaging studies of the Stroop task (Haupt et al., 2009; Melcher \& Gruber, 2006, 2009; Aarts, Roelofs, \& van Turennout, 2008; Fan, Hof, Guise, Fossella, \& Posner, 2008; Liu et al., 2008; Roberts \& Hall, 2008; Carter \& van Veen, 2007; Kerns, 2006; van Veen \& Carter, 2005; Kerns et al., 2004; Fan et al., 2003; Peterson et al., 2002). Responses in the head of the left caudate also interested us. Stimulation of this region induces perseveration in picture naming (Gil Robles, Gatignol, Capelle, Mitchell, \& Duffau, 2005) and its activation increases when individuals translate rather than merely repeat words (Price, Green, \& von Studnitz, 1999), name pictures in competition from the more dominant language (Abutalebi et al., 2008), decide a letter string is a word in their second language when it is also a word with a different meaning in their first (van Heuven, Schriefers, Dijkstra, \& Hagoort, 2008), make a semantic judgment given a change in language (Crinion et al., 2006), or perceive a switch into a weaker second language in a narrative comprehension task (Abutalebi et al., 2007). Yet, surprisingly, this subcortical structure is not typically reported in functional studies of the Stroop task. We anticipated that our design might reveal its presence and so contribute to the development of an integrated account of caudate function.

\section{METHODS}

The study was approved by the National Hospital and Institute of Neurology's joint ethics committee.

\section{Participants}

Participants were 13 right-handed monolingual volunteers ( 6 men and 7 women). Those who fitted the monolingual criteria had a mean age of 47 years (range $=23-62$ years). Because interference on the Stroop task can be modulated by age (Bugg, DeLosh, Davalos, \& Davis, 2007), we were aware that the wide age range of our participants might decrease sensitivity to effects at the group level. However, a wide age range will not result in false positives and the effect of age on Stroop interference was not the focus of this study. Prior to data acquisition, all participants gave 
written informed consent and filled in a questionnaire to confirm that they were monolingual with English as their native language, had no history of difficulties learning to speak or read, and were MRI compatible with normal or corrected-to-normal vision.

\section{Experimental Design}

\section{Stroop Task}

Participants made a left or right keypress (using their index or middle finger on their right hand, respectively) to indicate whether the color of the visual stimulus was blue or pink. The stimulus was either the written word "PINK," the written word "BLUE," or a string of four XXXXs pre- sented either in the hue pink or blue at the center of the screen. The visual angle of each stimulus was $4.45^{\circ} \times 1.38^{\circ}$. The colors had identical saturation and luminance. The manual response had the advantage of allowing us to collect accurate and precise RT data in the noisy fMRI scanning environment and to minimize head motion which typically increases when the task is overt speech production.

We presented three types of trial: (1) congruent (C) when the color of the ink was the same as the word (e.g., the word BLUE in blue ink); (2) incongruent (I) when the color of the ink was different to the word (e.g., the word PINK in blue ink); and (3) neutral (N) when the letter string was a string of Xs (XXXX) in either blue or pink. Trial type only changed after a run of four or six trials of the previous type (see Figure 2), allowing us to dissociate the
Figure 2. Examples of stimulus runs, trials, and types. This figure needs to be viewed in color to appreciate the congruency and incongruency between the words and their color. The columns provided the following information: run number with four to six stimuli; trial number within the run; stimulus on the screen; response $=$ left for blue hue and right for pink hue; change $=$ change in hue $(+=$ yes there is a change); type of condition; switch between conditions where $\mathrm{nC}=$ event where trial type switched from neutral (n) to congruent $(\mathrm{C}), \mathrm{cI}=$ event where trial type switched from congruent (c) to incongruent (I), iN = event where trial type switched from incongruent (i) to neutral $(\mathrm{N}), \mathrm{nI}=$ event where trial type switched from neutral (n) to incongruent (I), $\mathrm{iC}=$ event where trial type switched from incongruent (i) to congruent $(\mathrm{C})$, and $\mathrm{cN}=$ event where trial type switched from congruent (c) to neutral $(\mathrm{N})$. Uppercase $=$ the event, lower case $=$ the previous trial type. Conflict change occurs at the onset or offset of incongruent trials. At the onset of incongruent trials, activation related to conflict and control increases. At the offset of incongruent trials, activation related to conflict and control decreases.

\begin{tabular}{|c|c|c|c|c|c|c|c|c|}
\hline \multicolumn{2}{|c|}{ Run Trial } & \multicolumn{4}{|c|}{ Stimulus Response Change Type } & \multicolumn{3}{|c|}{ Switch Conflict Activation } \\
\hline 1 & 1 & $X X X X$ & Left & & Neutral & & & \\
\hline 1 & 2 & $x x x x$ & Right & + & Neutral & & & \\
\hline 1 & 3 & $x x x x$ & Right & & Neutral & & & \\
\hline 1 & 4 & $x x x x$ & Right & & Neutral & & & \\
\hline 1 & 5 & $X X X X$ & Left & + & Neutral & & & \\
\hline 1 & 6 & $x X X X$ & Left & & Neutral & & & \\
\hline 2 & 7 & BLUE & Left & & Congruent & $\mathrm{nC}$ & & \\
\hline 2 & 8 & PINK & Right & + & Congruent & & & \\
\hline 2 & 9 & PINK & Right & & Congruent & & & \\
\hline 2 & 10 & BLUE & Left & + & Congruent & & & \\
\hline 3 & 1 & PINK & Left & & Incongruent & cI & ONSET & 不 \\
\hline 3 & 2 & PINK & Left & & Incongruent & & & \\
\hline 3 & 3 & BLUE & Right & + & Incongruent & & & \\
\hline 3 & 4 & BLUE & Right & & Incongruent & & & \\
\hline 3 & 5 & PINK & Left & + & Incongruent & & & \\
\hline 3 & 6 & BLUE & Right & + & Incongruent & & & \\
\hline 4 & 7 & $x x x x$ & Right & & Neutral & iN & OFFSET & 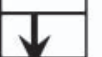 \\
\hline 4 & 8 & $x x x x$ & Right & & Neutral & & & \\
\hline 4 & 9 & $x x x x$ & Left & + & Neutral & & & \\
\hline 4 & 10 & $x x x x$ & Left & & Neutral & & & \\
\hline 5 & 1 & PINK & Left & & Incongruent & nI & ONSET & 木 \\
\hline 5 & 2 & BLUE & Right & + & Incongruent & & & \\
\hline 5 & 3 & BLUE & Right & & Incongruent & & & \\
\hline 5 & 4 & PINK & Left & + & Incongruent & & & \\
\hline 6 & 5 & BLUE & Left & & Congruent & $\mathrm{iC}$ & OFFSET & 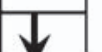 \\
\hline 6 & 6 & BLUE & Left & & Congruent & & & \\
\hline 6 & 7 & PINK & Right & + & Congruent & & & \\
\hline 6 & 8 & BLUE & Left & + & Congruent & & & \\
\hline 6 & 9 & PINK & Right & + & Congruent & & & \\
\hline 6 & 10 & PINK & Right & & Congruent & & & \\
\hline 7 & 1 & $x x x x$ & Right & & Neutral & $\mathbf{c N}$ & & \\
\hline 7 & 2 & $x x x x$ & Right & & Neutral & & & \\
\hline 7 & 3 & $x x x x$ & Left & + & Neutral & & & \\
\hline etc & etc & etc & etc & etc & etc & etc & etc & etc \\
\hline
\end{tabular}


onset of activation for each condition (see below for more details).

To dissociate the effects of incongruency from the effects of response change, a change in stimulus color never coincided with a change in the type of trial (see Figure 2). To fully control the position of response change within each run of trials, we categorized events in runs of 10 trials. Each run involved four stimuli of one type followed by six stimuli of another type (see Figure 2). This way, switches in trial type were always in the same position (i.e., first and fifth position or first and seventh position) in a run of 10 trials, but the type of trial switch was not predictable. Color changes then occurred randomly at all other positions (i.e., 2, 3, 4, 5/6, 7, 8, 9) with each color change position sampled the same number of times for each trial type. Subjects could therefore not successfully predict the color of the next stimulus or the required response. Nevertheless, by using short runs of the same type of trial (incongruent, congruent, or neutral), subjects would be able to predict, to some extent, the degree of interference on the next trial during the sustained part of a run. Consequently, the implementation of mechanisms that control interference may be partly under conscious/strategic influence.

\section{Simon Task}

The Simon task was essentially the same as the Stroop task, except that word stimuli were replaced with rectangles of a single color (either pink or blue) presented on the right, left, top, or bottom of fixation. Luminance and saturation of the blue and pink matched the values of the Stroop task. The rectangles matched the size of the words in the Stroop task (i.e., $4.45^{\circ} \times 1.38^{\circ}$ ) but were positioned equidistant $\left(4.4^{\circ}\right)$ from the central fixation point (whereas all stimuli in the Stroop task were presented at the center of the screen). Trial congruency was determined by the hemifield of the stimulus. For instance, a pink stimulus (requiring a right keypress) presented right of fixation was a congruent trial. The same stimulus presented left of fixation was an incongruent trial. Neutral trials were colored rectangles presented above or below fixation. All the timing, blocking, and ordering of conditions were identical to those used in the Stroop task.

\section{Procedure}

Stroop and Simon tasks alternated in different but consecutive scanning sessions, each of which lasted 4 min $32 \mathrm{sec}$ and consisted of 180 trials, 35 of which involved a change in trial type. In each scanning session, half the trials (90) were pink stimuli, the other half were blue stimuli. Stimulus onset asynchrony was 1440 msec comprising fixation "+" (400 msec), a blank screen (100 msec), stimulus presentation (630 msec), and another blank screen (310 msec). This allowed $940 \mathrm{msec}$ for the participants to respond (as fast and accurately as possible) from the onset of the stimulus to the beginning of the next

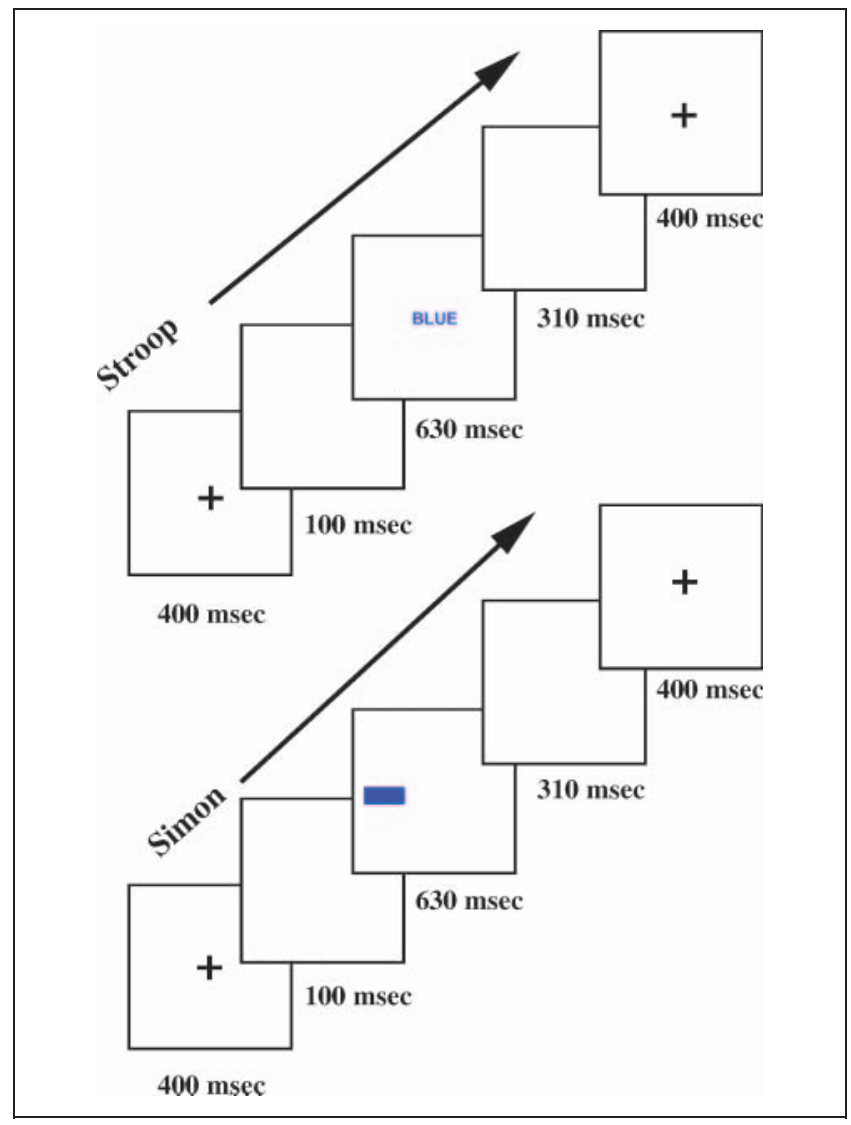

Figure 3. Illustration of Stroop and Simon paradigms. The sequence of events for each trial involved fixation for $400 \mathrm{msec}$, followed by a blank screen for $100 \mathrm{msec}$, the stimulus for $630 \mathrm{msec}$, another blank screen for $310 \mathrm{msec}$.

trial (see Figure 3). The fast presentation rate of the stimuli was chosen to keep the subjects engaged in the task and maximize the hemodynamic response to each condition (Friston, Zarahn, Josephs, Henson, \& Dale, 1999). We therefore do not attempt to dissociate responses to successive trials within a block. Instead, we either focus on block effects or on activation related to condition changes that occurred every four to six trials (5760$8640 \mathrm{msec}$ ) with at least twice as long between changes of the same type (e.g., cI changes were followed by iN or iC which in turn would be followed by $\mathrm{nC}, \mathrm{nI}, \mathrm{cN}$ or $\mathrm{cI}$ ).

Twelve participants completed between four and eight sessions (half Stroop and half Simon), depending on time or fatigue, with the 13 th subject only completing two sessions. The number of sessions completed does not affect the proportion of trials for each event type because all conditions were fully counterbalanced within each session. Within each session, there were seven runs of each condition (I, C, or N) with eight switches between incongruent and congruent (cI or $\mathrm{iC}$ ) and 21 trials of each condition that were preceded and followed by the same condition (iIi, cCc, nNn). Over sessions, this resulted in a minimum of 16,24 , or 32 trials per effect of interest (depending on whether the subject completed 4, 6, or 
8 sessions). With 13 subjects, we had sufficient sensitivity to (a) replicate previous effects and (b) find new effects of interest. Therefore, we did not include more subjects.

\section{Data Acquisition}

A Siemens 1.5-T Sonata scanner was used to acquire both anatomical and functional images from all participants. Anatomical T1-weighted images were acquired using a three-dimensional (3-D) MDEFT (modified driven equilibrium Fourier transform) sequence and 176 sagittal partitions with an image matrix of $256 \times 224$ and a final resolution of $1 \mathrm{~mm}^{3}$ [repetition time (TR)/echo time (TE)/ inversion time (TI), 12.24/3.56/530 msec].

Functional T2*-weighted echo-planar images with BOLD contrast comprised 35 axial slices of $2 \mathrm{~mm}$ thickness with $1 \mathrm{~mm}$ slice interval and $3 \times 3 \mathrm{~mm}$ in-plane resolution. Ninety volumes were acquired per session. Effective TR was $3.15 \mathrm{sec} /$ volume, $\mathrm{TE}=50 \mathrm{msec}$, flip angle $=90^{\circ}$. TR and stimulus onset asynchrony did not match, allowing for distributed sampling of slice acquisition across the experiment (Veltman, Mechelli, Friston, \& Price, 2002), which provides implicit "jittering." To avoid Nyquist ghost artifacts, a generalized reconstruction algorithm was used for data processing. After reconstruction, the first four volumes of each session were discarded to allow for T1 equilibration effects.

\section{fMRI Data Analysis}

Data preprocessing and the statistical analyses were performed using algorithms implemented in SPM5 (Wellcome Trust Centre for Neuroimaging, London, UK). Spatial preprocessing includes realignment with unwarping (to correct for head motion), nonlinear spatial normalization to the Montreal Neurological Institute (MNI) 152 space (with conversion to $2 \mathrm{~mm}^{3}$ voxels), and spatial smoothing (isotropic $8 \mathrm{~mm} \mathrm{FWHM).}$

Our choice of first- and second-level statistical analyses was constrained by the poor temporal resolution of fMRI. The slow hemodynamic response makes it difficult to dissociate the response of one trial from the next in the way that is possible in RT data. This problem would have been reduced if we had used a longer interstimulus interval but this would have (i) reduced the efficiency of our design to all effects (Friston et al., 1999); (ii) made it more difficult to maintain the attention of our participants because the task would have been very unengaging; and (iii) with longer interstimulus intervals, RTs would have been longer and more variable across conditions. Critically, however, we can identify all our effects of interest, within our fast presentation design, by looking at how activation changed with a switch in condition rather than by looking at how activation changed within a run of the same condition. Thus, the onset of interference can be identified as an activation increase for an incongruent trial that follows a run of congruent or neutral trials. Con- versely, the control of interference can be identified as higher activation at the end of a run of incongruent trials than at the onset of the next condition. This is equivalent to decreased activation when a run of incongruent trials switched to a run of congruent or neutral trials but not when a run of neutral trials switched to a run of congruent trials (i.e., decreased activation is specific to the incongruency context; see Figure 1). In addition, we validate our results by comparing activation during a run of sustained incongruency (i.e., all incongruent trials minus the onset trial) compared to sustained congruency or sustained neutral trials. On the basis of this rationale, our firstand second-level statistical analyses aimed to separate the first trial of a run (which we refer to as the onset trial) from the subsequent trials of that condition (which we refer to as the sustained trials).

First-level statistical analyses, performed at an individualsubject level, used a least square regression analysis. Low-frequency noise and signal drift were removed with high-pass filtering (1/128 Hz cutoff) and residual temporal autocorrelation were approximated by an AR(1) model and removed. We used an event-related analysis for all trials without modeling epochs/block effects (see Mechelli, Henson, Price, \& Friston, 2003 for justification and details). There were 11 regressors that were each convolved with a canonical hemodynamic response function. Nine modeled the three types of trials (I, C, N) in each of three contexts (i.e., preceded by i, c, or n). The 10th modeled color changes that signal response changes; and the 11th modeled errors (where the response did not match the color). By modeling out errors, our analysis focused on correct trials only when interference was successfully controlled. Onset trials were those when a condition was preceded by a different condition (iC, $\mathrm{cI}, \mathrm{nI}, \mathrm{nC}, \mathrm{iN}, \mathrm{cN}$ ). Sustained trials were those when the condition was preceded by the same type of trials (iI, $\mathrm{cC}, \mathrm{nN}$ ). Our event-related analysis was therefore able to segregate switch trials from sustained trials in a fully balanced factorial design that modeled the three different conditions in the three different contexts.

From the first-level analysis, we computed the nine contrasts for both the Stroop and Simon tasks (i.e., a total of 18 contrasts). The nine contrasts included the six types of condition switch (cI, cN, iC, iN, nI, nC), the response change $(\mathrm{R})$, the comparison of all incongruent sustained trials to all neutral sustained trials (i.e., ii $>\mathrm{nn}$ ), and all congruent sustained trials to all neutral sustained trials (i.e., $c c>n n$ ).

At the second level, we report the results of three different analyses of variance (ANOVA) each with nonsphericity corrections in SPM5.

\section{Analysis 1: Stroop Interference Change Trials}

This analysis included the seven switch conditions from the Stroop task only: the six contrasts modeling a switch in Stroop condition (cI, cN, iC, iN, nI, nC) and the response 
change (R). No data from the sustained trials (cC, iI, nN) were included. The contrasts modeling switch trials are estimates of the degree to which activation changed on the switch trial relative to preceding trials, after modeling out the effect of all other regressors.

The effects of interest in Analysis 1 were the activation changes associated with a switch between incongruent and congruent trials ( $\mathrm{cI}$ and $\mathrm{iC}$ ). Thus, the onset of interference and its control was measured as an activation increase for condition $[\mathrm{CI}]$ when the first incongruent trial followed a run of congruent trials. Conversely, the effect of interference and its control at the end of an incongruent trial was measured as an activation decrease for condition $[\mathrm{iC}]$ when a run of incongruent trials changed to a run of congruent trials (see Figure 1). As explained in the Introduction, the effect of interference was expected to be greater at the start of an incongruent run, whereas activation related to control mechanisms was expected to be greater at the end of an incongruent run. We can also distinguish the control mechanisms that actively suppress word interference from the effect of inhibiting task-irrelevant reading because activation related to the control mechanisms was expected to be greater at the end of a run of incongruent trials, whereas the effect of word inhibition was expected to reduce activation at the end of a run of incongruent trials (Harrison et al., 2005).

The main focus of this first analysis was on the switches between congruent and incongruent trials because this controlled for the effect of word recognition and, therefore, provided a more sensitive measure than the switch between incongruent and neutral trials (which is confounded by word recognition differences). Nevertheless, by including all other switch conditions (cN, iN, nI, nC and $\mathrm{R}$ ) in the same analysis, we were able to ensure that our effects were specific to incongruency and to identify and compare these effects to those of response change and lexicality change [i.e., the switch from neutral to congruent (nC) or neutral to incongruent (cI)]. Analysis 3 ensured that any effect of incongruent versus congruent was also present for incongruent versus neutral.

\section{Analysis 2: Stroop versus Simon Effects on Interference Change Trials}

This analysis was identical to Analysis 1 except that we added the seven corresponding contrasts from the Simon task. This resulted in 14 contrast images that allowed us to compare the control of word interference (Stroop) to the control of spatial interference (Simon).

\section{fMRI Analysis 3: Stroop versus Simon Analysis of Sustained Trials}

To validate the findings of Analyses 1 and 2, our third analysis focused on the effect of sustained incongruency. If the areas activated at the end of a run of incongruent Stroop trials reflect the control of word interference, then we would also predict that the same regions should also be more activated for sustained incongruent trials (= all but the onset trial) than sustained congruent trials or sustained neutral trials. In order to look at the critical interaction of task and the difference between sustained incongruent and congruent trials, our third ANOVA included four contrasts from the first level: Stroop incongruency sustained trials $>$ Stroop neutral sustained trials (ii-nn); Stroop congruency sustained trials $>$ Stroop neutral sustained trials (cc-nn); Simon incongruency sustained trials $>$ Simon neutral sustained trials (ii-nn); and Simon congruency sustained trials $>$ Simon neutral sustained trials(cc-nn). Note, however, that this analysis is less sensitive to the analysis of switch trials because it sums over the order of sustained trials (Trials 2, 3, 4, 5, 6); therefore, effects that increase or decrease across a run of trials will be treated as error.

\section{Statistical Thresholds}

In Analysis 1, we report regions where activation was significant at $p<.05$ after correction for multiple comparisons across the whole brain either in height $(Z=4.8)$ or extent $(>120$ voxels at $p<.001)$. In addition, we lowered the threshold to $p<.001$, uncorrected in both Analyses 1 and 2 to explore the pattern of effects in the anterior cingulate and inferior frontal cortices previously associated with incongruency in the Stroop paradigm (e.g., Fan et al., 2003). In Analysis 3, we focus only on the regions identified in Analyses 1 and 2 .

\section{Analysis of In-scanner Behavior}

We ran five different behavior analyses on both accuracy and RTs. All involved separate repeated measures ANOVAs in SPSS, for RTs and for accuracy. The results confirmed that our effects were consistent with the previous literature. Full details are provided in the Appendix. In the main text, we report the results of two different analyses that correspond, as closely as possible, to those conducted on the functional imaging data. One focused on the first versus last trial of incongruent runs relative to the first versus last trial of congruent runs with three variables: trial type (incongruent/congruent), task (Stroop/Simon), and context (first vs. last trial of a run). The other analysis focused on sustained incongruency relative to both sustained congruency and sustained neutral trials with two variables: task (Stroop/Simon) and trial type (incongruent/congruent/ neutral) averaged over the sustained trials (Positions 2-6) and not including the first onset trial.

\section{RESULTS}

\section{Behavioral Responses during Scanning}

Here we report only the effects that are relevant for interpreting the functional imaging data. All other effects and 
details are reported in the Appendix and illustrated in Figure 4. As expected, responses to incongruent trials were less accurate and slower than those to congruent trials in all analyses (see Figure 4 and the Appendix for details), even though we used a manual version of the Stroop with only two colors. The most important finding was that responses were least accurate for the first relative to last trial in an incongruent run, and this was confirmed by a significant interaction between incongruency (incongruent $>$ congruent) and context (last $>$ first trial of a run) across tasks with no significant $(F<1)$ threeway interaction (Task $\times$ Incongruency $\times$ Context). The mechanisms for controlling word interference were therefore more effective at the end of a run of incongruent trials (i.e., the last trial) than at the start (i.e., the first trial). Moreover, the consistency of this effect across task demonstrates the same pattern of effects for word interference in the Stroop task and spatial interference in the Simon task.

\section{fMRI Analyses}

Below, we report the results of three analyses. The first analysis focused on the Stroop task and identified the main effects of interference change, lexicality change, and response change. The second analysis compared the effect of interference change in the Stroop and Simon task. In both the first and second analysis, interference change was identified when congruent trials changed to incongruent trials or vice versa (see Methods for details). We then dissociated activation associated with conflict (i.e., greater at the onset of incongruency) from activation associated with control (i.e., greater at the end of a run of incongruent trials than the beginning of a run of incongruent trials; see Figure 1 for rationale). The third analysis compared the effect of sustained incongruent trials (when control should be highest) to sustained congruent trials and sustained neutral trials. This allowed us to test the predictions of Analyses 1 and 2, and compare the effect of sustained incongruency in the Stroop and Simon tasks.

\section{fMRI Analysis 1: Stroop Interference Change Trials}

Here we report the effects of: interference change, lexicality change, and response change. To do this, we only included the trials when there was a switch in condition. All trials without a change in condition (i.e., sustained trials) were excluded.

\section{The Effect of Interference Change}

The main effect of switching between incongruent and congruent trials reached a corrected level of significance in the left head of caudate and globus pallidus. Comparison of the size of this effect at the onset and end of interference indicated that it was driven by a fall in activation when incongruent trials changed to congruent trials (i.e., the end of interference) rather than an increase in activation when congruent trials changed to incongruent trials (i.e., the onset of interference, see Table 1 for statistical details). This is consistent with activation being higher at the end of a run of incongruent trials (see Analysis 3 for verification), consistent with the pattern of response that we predicted for the regions controlling word interference (i.e., higher at the end than at the start of an incongruent run). There was no corresponding effect in the right caudate, even when the statistical threshold was reduced to $p<.05$.

In our regions of interest (using a lower statistical threshold of $p<.001$, uncorrected), a ventral region of the left anterior cingulate showed a similar effect of incongruency
Figure 4. In-scanner behavioral data. RTs (msec) and accuracy (\%) for congruent (open squares) and incongruent (triangles) trials across each of the six serial positions in a run of one type of trial (see Figure 2). For further details on means and standard errors, see Appendix.

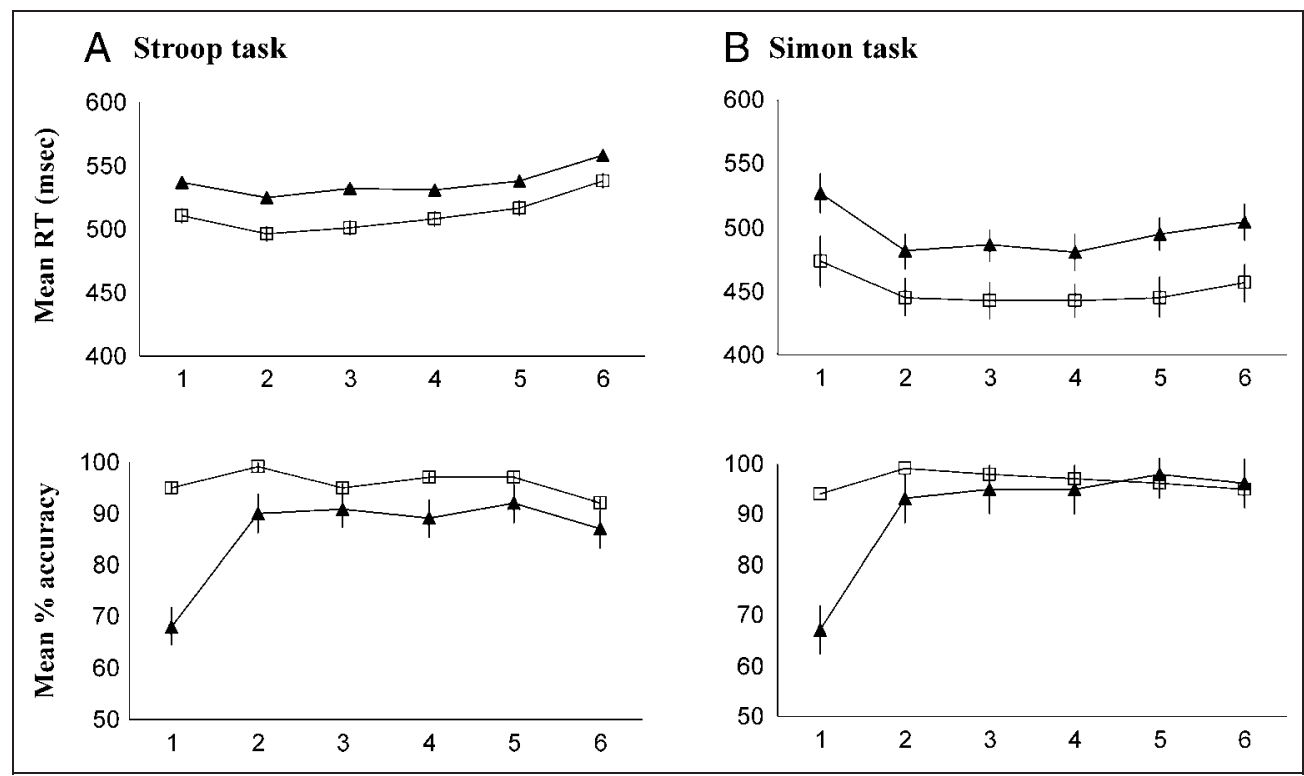


Table 1. The Effect of the Onset and End of Word Interference

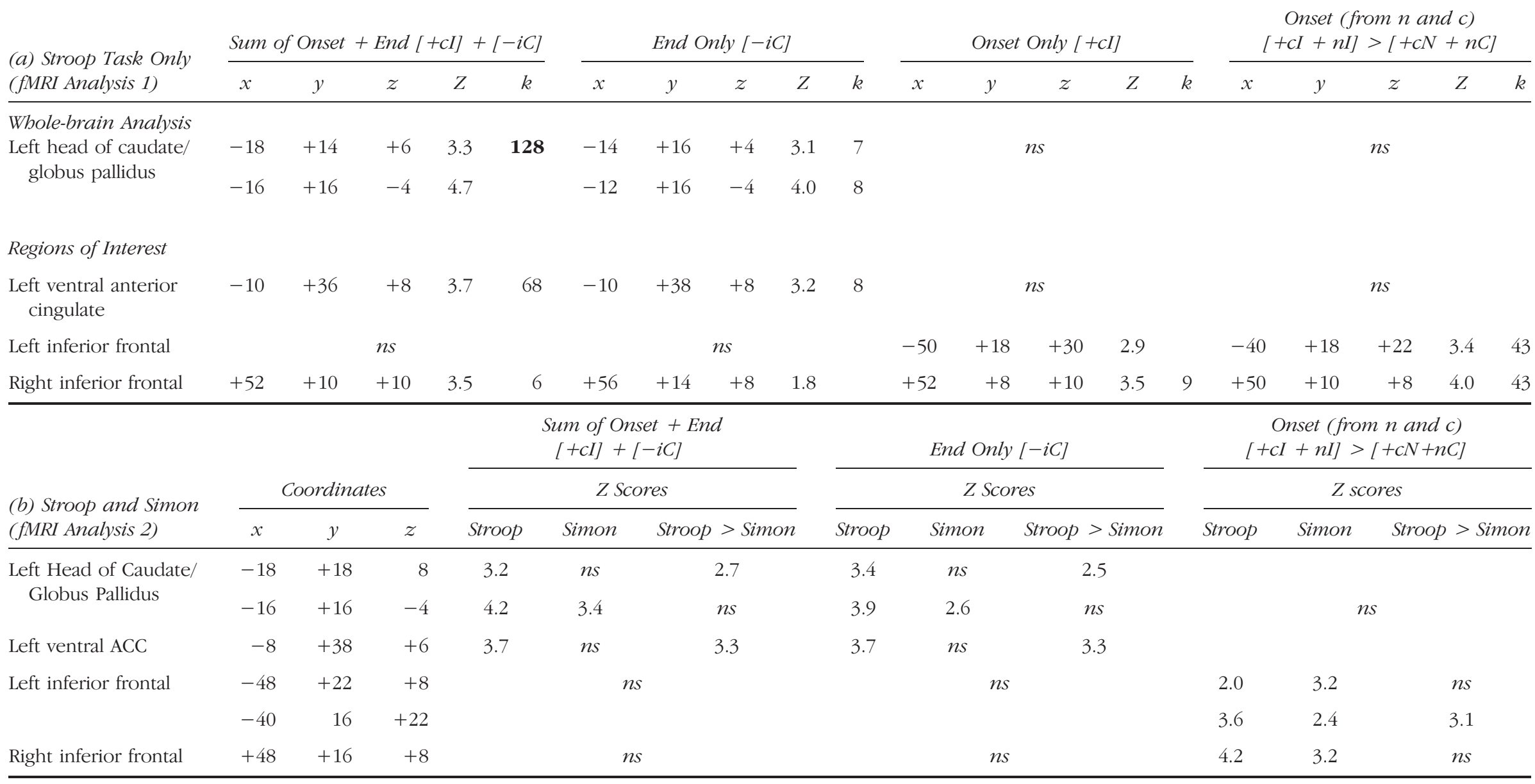

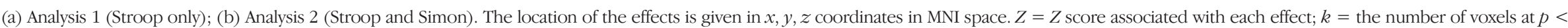

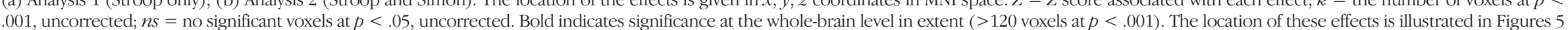
and 6. In the contrast description (top row), uppercase letters refer to events (Congruent or Incongruent) and lowercase letters refer to the previous trial (either congruent, incongruent, or $n$ eutral). 
to that observed in the left head of caudate, that is, a greater activation change at the end of word interference relative to its onset (see Table 1). In contrast, right and left inferior frontal activation was higher at the onset of word interference relative to all other conditions (see Table 1).

\section{The Effect of Lexicality Change}

A switch from neutral to congruent or incongruent (i.e., words $>$ XXXXs) activated left occipito-temporal cortex associated with written word processing (61 voxels at $p<.001$, uncorrected with MNI peaks at $x=-42, y=$ $-58, z=-18, Z$ score $=3.9$; and at $x=-38, y=$ $-44, z=-22, Z$ score $=3.4)$. Although this effect is not the focus of our article, it illustrates that word recognition processes were active during the Stroop task.

\section{The Effect of Response Change}

Response changes (indicated by a change in stimulus color) activated dorsal anterior cingulate cortex, along with the supplementary motor area (SMA) as well as left and right dorsal supramarginal gyri, sensorimotor cortex, and putamen (see Table 2).

\section{fMRI Analysis 2: The Effect of Interference Change in the Stroop versus Simon Tasks}

The aim of this analysis was to determine whether the effects of incongruency that we observed in the Stroop

Table 2. The Effect of Response Change during the Stroop Task

\begin{tabular}{lcccccc}
\hline Region & Hem & \multicolumn{1}{c}{$x$} & $y$ & $z$ & $Z$ & $k$ \\
\hline Dorsal cingulate & $\mathrm{M}$ & +4 & +4 & +48 & 4.4 & 588 \\
& $\mathrm{M}$ & +2 & -8 & +42 & 4.1 & \\
SMA & $\mathrm{M}$ & +4 & 0 & +58 & 4.4 & \\
& $\mathrm{M}$ & 0 & -4 & +64 & 4.4 & \\
Sensorimotor & $\mathrm{L}$ & -28 & -18 & +60 & 5.1 & 287 \\
& $\mathrm{~L}$ & -28 & -4 & +58 & 4.8 & \\
& $\mathrm{R}$ & +36 & -4 & +56 & 4.9 & 112 \\
Supramarginal & $\mathrm{L}$ & -44 & -36 & +46 & 4.0 & 143 \\
& $\mathrm{~L}$ & -44 & -42 & +52 & 3.6 & \\
& $\mathrm{R}$ & +42 & -36 & +44 & 4.0 & 58 \\
Putamen & $\mathrm{L}$ & -18 & -6 & +12 & 4.3 & 48 \\
& $\mathrm{R}$ & +26 & 0 & +12 & 4.1 & 57 \\
\hline
\end{tabular}

The effects of response change in the Stroop analysis are given in $x, y, z$ coordinates in MNI space. Hem $=$ hemisphere; $\mathrm{L}=$ left hemisphere; $\mathrm{R}=$ right hemisphere; $\mathrm{M}=$ midline; $Z=Z$ score associated with each effect; $k=$ the number of voxels at $p<.001$, uncorrected; $n s=$ no significant voxels at $p<.05$, uncorrected. analysis were also observed in the Simon task. If the effects are common to both tasks, then they may reflect a general process that controls interference. Therefore, we focus our analysis of the Simon task on regions showing an effect of incongruency in the Stroop analysis reported above (i.e., left head of caudate, left globus pallidus, left ventral anterior cingulate, and bilateral inferior frontal cortices).

In the left head of caudate and the left ventral anterior cingulate (areas associated with suppressing word interference in the Stroop task), there was no corresponding effect in the Simon task $(Z<1.64 ; p>.05)$, hence, the effect was greater for suppressing word interference in the Stroop task than suppressing spatial interference in the Simon task (see Table 1). The locations of the left head of caudate and left ventral anterior cingulate effects are illustrated in yellow on the left side of Figure 5A. The fall in activation when incongruent Stroop trials changed to congruent Stroop trials (i.e., marking the end of word interference) is shown in Figure 5B.

In contrast to the word-specific effects in the left head of caudate and left ventral anterior cingulate, there were common effects of control for both tasks in the left globus pallidus (see Table 1) consistent with this region playing a role in controlling interference in both the Stroop and Simon tasks. The location of the effect in the left globus pallidus is shown in red in Figure 5A.

Finally, the onset of incongruency in the Simon task activated the left and right inferior frontal cortices, as in the Stroop task, although left prefrontal activation was stronger in the Stroop than in the Simon task (see Table 1). The top row of Figure 6 illustrates prefrontal activation at the onset of incongruency for both the Stroop and Simon tasks. The bottom row shows the relative activation for each change in trial type in the prefrontal regions. Activation was highest when trial type changed from congruent to incongruent (cI) and neutral to incongruent (nI) relative to all other trial and response type changes.

To summarize, by comparing activation at the onset and end of interference in the Stroop and Simon tasks, we have shown that activation in (1) the left head of caudate and a ventral region of the left anterior cingulate is associated with the control of word interference in the Stroop task but was not detected for the control of spatial interference in the Simon task; (2) the left globus pallidus is involved in the control of both word and spatial interference; (3) prefrontal regions are involved in the onset of interference when errors are highest (and control is lowest); and (4) a more dorsal region of the anterior cingulate and the left putamen (amongst other areas) are involved in manual response change.

\section{fMRI Analysis 3: The Effect of Sustained Interference in the Stroop versus Simon Task}

The results reported above predict that activation in the left head of caudate and ventral anterior cingulate should 
be greater for sustained incongruent trials than sustained congruent trials or sustained neutral trials. Moreover, this effect should be present in the Stroop but not in the Simon task. We therefore conducted an analysis of the sustained trials (excluding the first onset trials) which had four contrasts from the first level: Stroop incongruency sustained trials $>$ Stroop neutral sustained trials (ii-nn); Stroop congruency sustained trials $>$ Stroop neutral sustained trials (cc-nn); Simon incongruency sustained trials $>$ Simon neutral sustained trials (ii-nn); Simon congruency sustained trials $>$ Simon neutral sustained trials (Cc-nn). This analysis of sustained trials is less sensitive than the analysis of switch trials (Analyses 1 and 2) because it sums over the order of sustained trials (Trials 2, 3, 4, 5, 6) and, therefore, effects that increase or decrease across a run of trials will be treated as error. Nevertheless, in a whole-brain analysis, the most significant interaction of interest (sustained incongruentsustained congruent for Stroop vs. Simon) was located in the left head of caudate (MNI coordinates: $x=-16, y=$ $+8, z=+12, Z=3.0, p=.001$; see Figure 5 C). No other

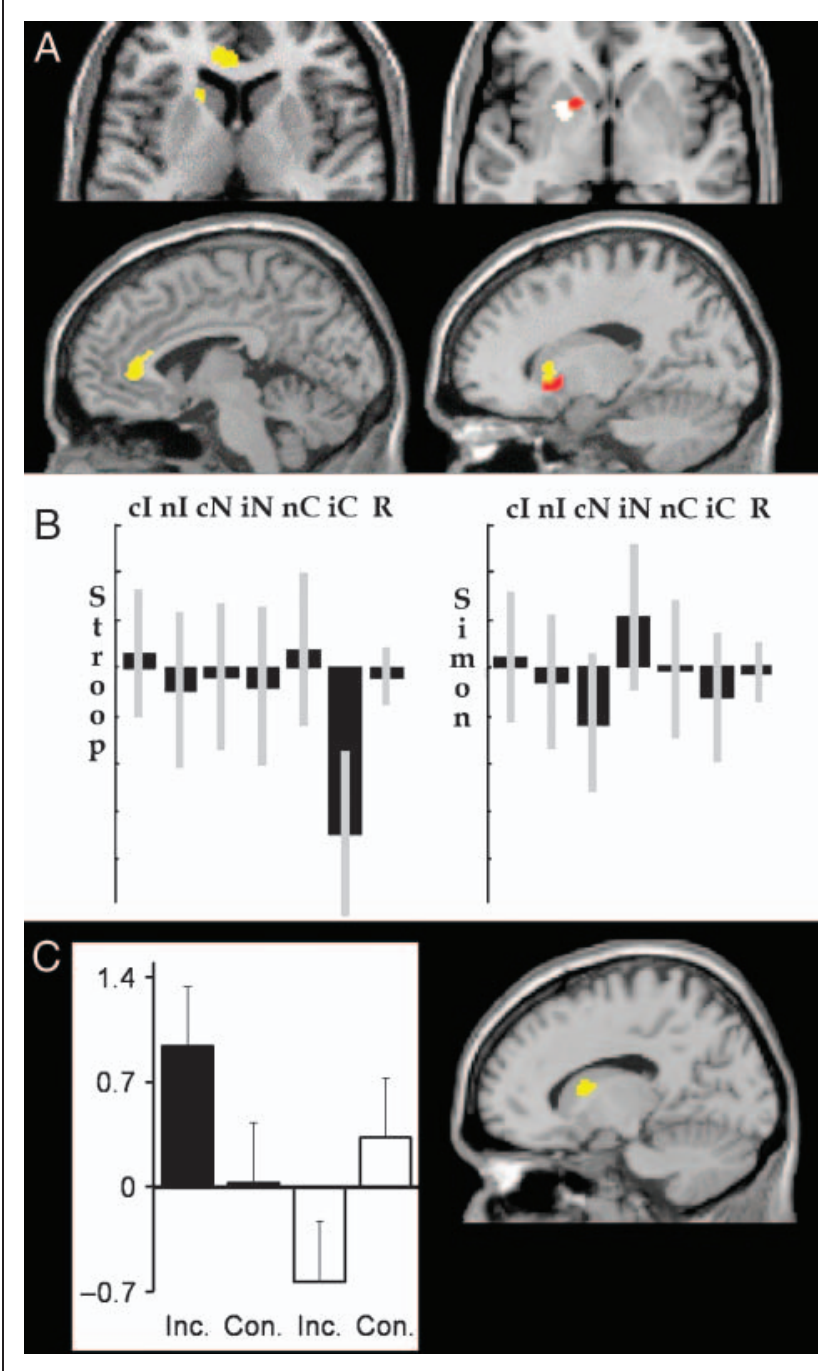

brain regions showed a corresponding effect at $p<.001$, uncorrected. When we reduced the threshold to $p<.05$, uncorrected, this interaction was still not significant in the anterior cingulate area identified in Analyses 1 and 2 above.

\section{DISCUSSION}

In this study, we sought to identify activation related to the control of word interference during the Stroop task. Participants were instructed to decide if the written words PINK or BLUE were presented in the color pink or blue. Using a block design, there were three conditions: incongruent, when the word and its color were not the same (e.g., PINK in blue); congruent, when the word and its color were the same (e.g., PINK in pink); or neutral, when a string of four Xs was presented in either pink or blue (see Figure 2). As expected, word interference was greater during incongruent trials relative to congruent and neutral trials. This was illustrated by the consistently longer RTs to incongruent trials at both the beginning and end of a block. In addition, the error data indicate

Figure 5. Activation change for controlling interference. (A) The results from fMRI Analysis 2 that compared trials where there was a change in condition. Red and yellow indicate the location of activation related to the control of interference (i.e., higher at the end of a run of incongruent trials). The red area was involved in the control of interference in both the Stroop and the Simon tasks $(p<.01)$. The yellow area was involved in the control of interference in the Stroop task more than the Simon task. White indicates the location of activation related to response change during both the Stroop and Simon task $(p<.001)$. The axial slices are positioned at $z=+6$ (left) and $z=-2$ (right). The sagittal slices are positioned at $x=-4$ (left side of figure) and $x=-16$ (right side of figure). (B) Relative parameter estimates for activation change from one condition to another in the left head of caudate (at $x=-18, y=+18$, $z=8)$. $\mathrm{C}=$ congruent; $\mathrm{I}=$ incongruent; $\mathrm{N}=$ neutral; $\mathrm{c}=$ in the context of congruent; $\mathrm{i}=$ in the context of incongruent; $\mathrm{n}=$ in the context of neutral; $\mathrm{R}=$ response change. Bars on the mean of each estimate represent $90 \%$ confidence intervals. The scale is identical for both Stroop and Simon, and the effects were extracted from the same analysis (Analysis 2). The feature to note is the decrease in activation for $\mathrm{iC}$, as predicted for control in Figure 1 . We propose that the reason that this decrease was not observed for iN is there is no urgency to release word inhibition mechanisms when a neutral trial is presented but there is when a congruent trial is presented because word inhibition is potentially disadvantageous. (C) The results from fMRI Analysis 3 showing activation (parameter estimates) in the left head of caudate for sustained incongruency relative to sustained neutral trials (black) and sustained congruency relative to sustained neutral trials (MNI coordinates: $x=-14, y=+8$, $z=+12$ ). Inc. $=$ parameter estimates for incongruent sustained trials $>$ neutral sustained trials; Con. = parameter estimates for congruent sustained trials $>$ neutral sustained trials; black bars $=$ Stroop task (first 2 columns); white bars = Simon task (second two columns). The lines on each bar represent the standard error across subjects in the parameter estimate. The right-hand image shows the caudate activation on a sagittal slice positioned at $x=$ -14 (in MNI coordinates). 


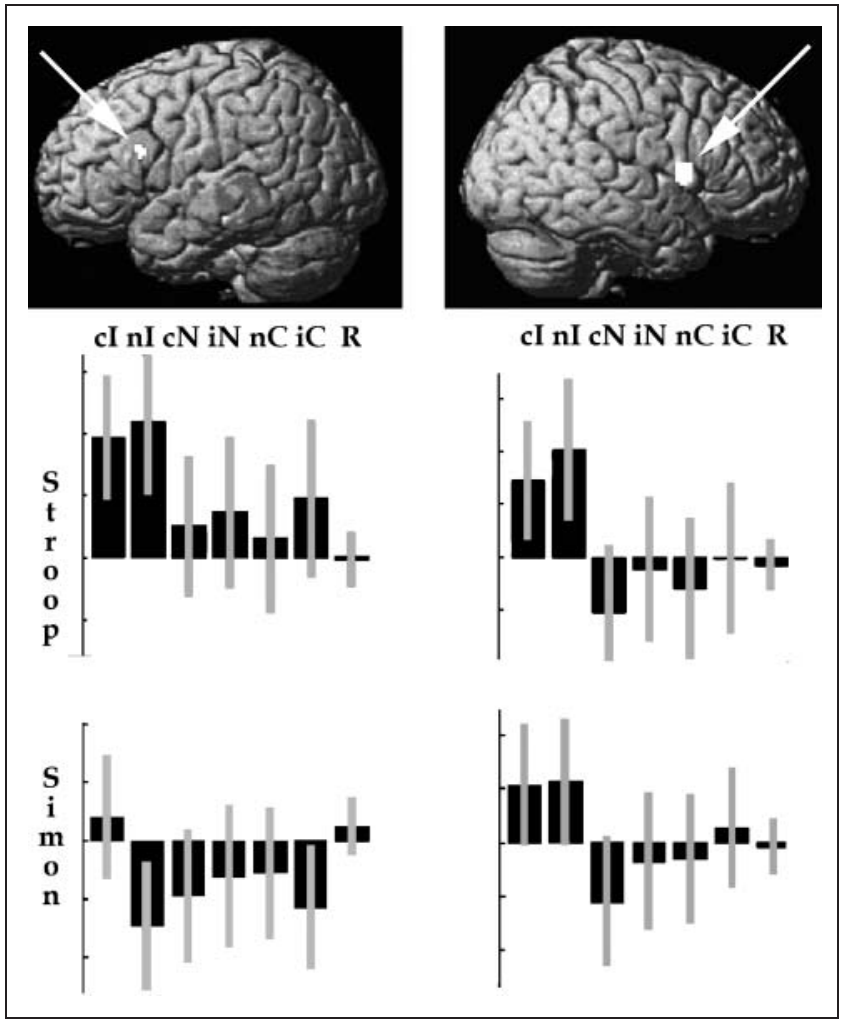

Figure 6. Activation change for the onset of interference. Top: Inferior frontal activation (in white) for the onset of incongruency rendered on the SPM canonical brain. Left hemisphere shown on left, and right hemisphere shown on right. Lower: The relative parameter estimates for each switch condition (Analysis 2) at the location of the effects shown directly above in the top row (i.e., left inferior frontal cortex on left, right inferior frontal cortex on right). $\mathrm{C}=$ congruent; $\mathrm{I}=$ incongruent; $\mathrm{N}=$ neutral; $\mathrm{c}=$ in the context of congruent; $\mathrm{i}=$ in the context of incongruent; $\mathrm{n}=$ in the context of neutral; $\mathrm{R}=$ response change. Lines on each bar represent $90 \%$ confidence intervals. The scale is identical for both Stroop and Simon tasks.

that the control of interference was least successful on the first incongruent trial of a block (see Figure 4). The behavioral data therefore supported our rationale that the brain areas involved in controlling interference would be more activated at the end relative to the start of a run of incongruent trials (see Figure 1).

Our results highlight the role of the left head of caudate in the control of written word interference during the Stroop task. Specifically, activation in the left head of caudate was consistent with the behavioral data. Like the RTs, left head of caudate activation was higher for sustained incongruent trials than sustained congruent or neutral trials (fMRI Analysis 3). Like the accuracy data, left head of caudate activation was higher at the end of a run of incongruent trials than at the start of incongruent trials (fMRI Analysis 1). These effects were also greater during the Stroop task than the Simon task (fMRI Analysis 2), which suggests that the left head of caudate is more involved in the control of word interference than the control of spatial interference. Below, we consider whether activation in the left head of caudate in the Stroop task is (a) specific to word inhibition; (b) involved in suppressing task-irrelevant information that is more interfering in the Stroop task than the Simon task; or (c) involved in amplifying task-relevant information (i.e., color processing; see Egner et al., 2007).

Our experimental manipulations also allow us to distinguish the response in the left head of caudate from that in several other areas including the left putamen, left globus pallidus, ventral anterior cingulate, dorsal anterior cingulate, and prefrontal regions. Our focus on the left head of caudate reflects (a) the significance of its response to interference change following a whole-brain search with a correction for multiple comparisons in fMRI Analysis 1; (b) the replication of this effect in the comparison of sustained incongruent to sustained congruent and neutral trials in fMRI Analysis 2; and (c) prior studies showing left head of caudate activation associated with language control in bilingual speakers combined with a lack of prior studies showing left caudate activation during the Stroop task. In contrast, activation of the left ventral anterior cingulate was only identified in fMRI Analysis 1, when we used a region-of-interest analysis based on prior knowledge from previous studies (e.g., Orr \& Weissman, 2009; Kerns et al., 2004). Moreover, we did not detect ventral anterior cingulate activation in fMRI Analysis 3, which compared sustained incongruent trials relative to sustained congruent and neutral trials. This suggests that, unlike left caudate activation, ventral anterior cingulate activation was not consistently high across all the sustained incongruency trials (Trials 26) when error rates and behavioral data showed good control of word interference. Below, we discuss the role of the left head of caudate in the suppression of word interference before turning to a discussion of the effects in other regions.

\section{Left Head of Caudate}

Previous studies have observed left head of caudate activation in the context of language-based conflict (e.g., Abutalebi et al., 2007, 2008; van Heuven et al., 2008; Crinion et al., 2006; Gil Robles et al., 2005) but leave open its precise functional role. Our results refine what is known about the role of the left head of caudate. They delimit the way it helps to ensure context-appropriate behavior (e.g., Chee, 2006) and generalize its function beyond selecting motor sequences for articulation (e.g., Friederici, 2006). Specifically, we propose that left head of caudate activation inhibits a plan triggered by the incongruent word rather than inhibiting the actual vocal response itself. This proposal follows from our observation that incongruency effects in the left head of caudate were observed in the context of a manual version of the Stroop task that did not require vocal responses.

Our proposal that the left head of caudate is involved in the inhibition of action plans is supported by the fMRI findings of Lungu, Binenstock, Pline, Yeaton, and Carey (2007). In their study, subjects performed an incongruent 
visual tracking task, which involved moving their finger in the opposite direction to a visual stimulus after practicing moving their finger in the same direction as the visual stimulus. These authors found that, in subjects whose performance improved over 30 sec of incongruent visual tracking, activation increased in the left head of caudate and decreased in prefrontal cortex. Lungu et al. (2007) proposed that their caudate activation could indicate the use of higher-order verbal rules such as "move finger in opposite direction." We also observed different patterns of effects in the frontal and left head of caudate regions using a Stroop task rather than a visual tracking task. However, an explanation in terms of higher-order verbal rules during the execution of a nonverbal task does not explain why we did not observe left head of caudate activation during the Simon task.

An alternative explanation for why left head of caudate activation was observed in the Stroop but not the Simon task is that this region may play a role in overcoming habitual or overlearnt actions (Parsons, Harrington, \& Rao, 2005; Shadmehr \& Holcomb, 1999). By this account, the left head of caudate is more involved in controlling word interference in the Stroop task than spatial interference in the Simon task because, prior to the experiment, a word is more strongly associated with its verbal response than a spatial location is associated with a response based on color. This account can therefore explain why we found greater left head of caudate activation in the Stroop than in the Simon task without advocating any verbally specific processing. It can also explain why error rates were higher for the onset of incongruency in the Stroop task relative to the Simon task.

The role of the left head of caudate in the control of overlearnt, nonverbal visuomotor tracking has been demonstrated by Shadmehr and Holcomb (1999). They showed that the left head of caudate was only activated when previously learnt movements had to be inhibited. Head of caudate activation has also been associated with efficient inhibitory control in overcoming a prepotent response in a stop-signal task (Li, Yan, Sinha, \& Lee, 2008). In line with the data of Li et al. (2008), Vink et al. (2005) suggest that the caudate nucleus becomes more active when subjects block an action from being automatically executed. Prior evidence therefore suggests that our results are best explained in terms of the left head of caudate playing a role in suppressing habitual action plans irrespective of whether they are verbal or nonverbal. In contrast, we did not find evidence for the alternative hypothesis (see above), that left head of caudate activation was involved in the amplification of color processing (Egner et al., 2007). This leads us to the conclusion that activation in the left head of caudate in our Stroop task reflected the suppression of task-irrelevant information that was more interfering in the Stroop task than the Simon task. Our supposition is that processes involved in suppressing word interference (whether in monolingual or bilingual speakers) are specializations of those in- volved in the control of action in general (e.g., Abutalebi \& Green, 2007; Green \& Price, 2001; Green, 1998).

Given prior evidence for the role of the left head of caudate in suppressing competing action plans, it is perhaps surprising that activation in this region has not previously been identified in functional imaging studies of the Stroop task. The most likely explanation for this inconsistency is that, contrary to previous studies, we used short runs of incongruent trials to segregate the onset of interference from sustained interference when control mechanisms are more likely to be implemented. In addition, we factored out activation related to a change in motor response. This allowed us to demonstrate that the incongruency effects in the left head of caudate were very close to the motor response change effects in the left putamen and so both effects may be lost to error variance if they are not modeled independently.

Notably, the head of caudate activation we observed was very left-lateralized with no corresponding effect in the right caudate, even when we reduced the statistical threshold to $p<.05$, uncorrected. Other studies have shown that the right head of caudate is involved in the early stages of reward related probability learning (Sharot, De Martino, \& Dolan, 2009; Delgado, Miller, Inati, \& Phelps, 2005) and bilateral head of caudate is involved in feedback learning in both neurologically normal participants (Tricomi \& Fiez, 2008; Tricomi, Delgado, McCandliss, McClelland, \& Fiez, 2006) and patients with Parkinson's disease (Shohamy, Myers, Kalanithi, \& Gluck, 2008; Shohamy, Myers, Onlaor, \& Gluck, 2004). Contrary to our study, however, these studies monitored changes over long experimental sessions, whereas our study showed effects in the left caudate over much shorter time periods ( $<15 \mathrm{sec})$. Further studies are required to investigate differences in the function of the left and right caudate. Nevertheless, there is a growing consensus across studies that left and/ or right caudate activation is required to learn a new response when a previously learned response is no longer rewarding (Shohamy et al., 2008).

\section{Other Effects}

A standard expectation is that prefrontal activation will increase in response to incongruity reflecting processes designed to decrease interference (e.g., Botvinick et al., 2001; see Roberts \& Hall, 2008, for a systematic review). Our results are in line with this expectation. We note in the present context that the bilateral inferior frontal activation we observed for incongruent Stroop trials is consistent with van Veen and Carter (2005), Fan et al. (2003), and Peterson et al. (2002). However, it is inconsistent with the data of Kerns et al. (2004), who reported right but not left frontal activation, and with that of Melcher and Gruber (2006), who found no prefrontal activation on incongruent Stroop trials. Interestingly, in our study, left and right inferior frontal activation was observed at 
the onset of interference in both the Stroop and Simon tasks, although more markedly for the Stroop task (Figure 6). The effects in the inferior frontal lobe regions may therefore be more consistent with a role in the early stages of controlling interference (Lungu et al., 2007).

Our second region of interest was the anterior cingulate. Here we dissociated contrasting contributions of the dorsal and ventral anterior cingulate during the Stroop task. The dorsal anterior cingulate was activated by a change in response, irrespective of the task. In contrast, left ventral anterior cingulate activation changed markedly at the end of a run of incongruent trials when the control of interference was high. Other studies have also dissociated the functions of different anterior cingulate regions (e.g., Orr \& Weissman, 2009; Aarts et al., 2008; Fan et al., 2008). Our study further contributes to the literature by showing that previous reports associating dorsal anterior cingulate with incongruency effects may have resulted from the conflation of incongruity effects with response change.

Finally, our results show an interesting dissociation in three different regions of the left basal ganglia: the head of caudate, putamen, and globus pallidus. The left head of caudate was more activated for the control of word interference than the control of spatial interference (see above). The left putamen activated when there was a response change in both the Stroop and Simon tasks. This is consistent with a study by Monchi, Petrides, Strafella, Worsley, and Doyon (2006), which implicate the caudate in planning a novel action and the putamen in its execution. Thirdly, the left globus pallidus was activated during the control of both word and spatial interference. This is interesting because the main output for the caudate and the putamen is via the globus pallidus, which then projects via the thalamus and the brainstem to motor cortex.

\section{Summary and Implications}

Our study highlights the role of the left head of caudate in word inhibition. Computational accounts of the Stroop task (e.g., Cohen et al., 1990) allow conflict to be resolved by amplifying target-relevant features (e.g., color) or suppressing distractor information (word) or both. Our data support the view that in a classic Stroop task, there is inhibition of the distractor. We do not suggest that the mechanisms controlling suppression are invariable. The precise way in which conflict is managed is likely to depend on the nature of the conflict. For instance, in a Stroop-like task involving the discrimination of actors versus political figures, when faces were the target and words the distracter, Egner and Hirsch (2005b) found amplification of neural response to face stimuli but no evidence of down-regulation when faces served as the distractor. Likewise, different kinds of conflict task afford different means for control. Whereas interference in the
Simon task can be controlled by blocking the effect of the spatial cue, interference cannot be controlled in this way in the Stroop task. In the Simon task, response conflict arises, at least initially, from the bias to respond according to the position of stimulus (e.g., left side-respond left). There is no verbally induced competing response. In the case of an incongruent stimulus, a correct response requires overcoming the effect of such automatic positional bias but this is not our focus of interest in the current study.

By comparing our findings to the previous literature, we are able to refine what we know about the role of the left head of caudate in word processing. Together, the results suggest that the left head of caudate is involved in controlling overlearnt competing action plans and is important, but not exclusive, to language control. Our study has, therefore, contributed novel insights into the role of the left head of caudate in word processing. Our data strongly motivate the extension of current accounts of the control of verbal interference (e.g., Fan et al., 2008; Egner \& Hirsch, 2005a, 2005b; Kerns et al., 2004) to include the caudate and other structures of the basal ganglia. Future studies are required to manipulate the level of overlearning prior to scanning and also to find a mechanistic account for how the different basal ganglia areas interact with one another and other frontal regions during word inhibition and language control. The contribution of our study is to highlight the role of the left head of caudate in these circuits.

\section{APPENDIX}

The group means (and standard errors) of the behavioral data plotted in Figure 4 are as follows:

\begin{tabular}{lccc}
\hline & Incongruent & Congruent & Neutral \\
\hline Reaction Times & (in Milliseconds & with Standard Errors) \\
Onset trials & & & \\
Stroop & $541(26)$ & $517(21)$ & $535(23)$ \\
Simon & $518(17)$ & $469(15)$ & $483(16)$ \\
Other trials & & & \\
Stroop & $537(21)$ & $512(18)$ & $505(17)$ \\
Simon & $489(14)$ & $446(13)$ & $467(14)$
\end{tabular}

Accuracy Data (in Percentages with Standard Errors)

Onset trials

\begin{tabular}{cccc} 
Stroop & $68(.04)$ & $94(.02)$ & $94(.01)$ \\
Simon & $73(.03)$ & $93(.02)$ & $93(.02)$ \\
Other trials & & & \\
Stroop & $90(.02)$ & $96(.01)$ & $95(.01)$ \\
Simon & $95(.01)$ & $97(.02)$ & $96(.01)$ \\
\hline
\end{tabular}




\section{Details of All Five Behavioral Analyses}

1. The Effect of Incongruency on the First versus Last Trial of a Run

In the analyses of incongruency (incongruent vs. congruent), task (Stroop vs. Simon), and context (first vs. last trial of a run), participants were less accurate and slower for incongruent trials than congruent trials. In the RT analysis, this main effect of incongruency $[F(1,12)=54.09, p<$ $.001]$ did not interact with either task or context $[F(1$, $12)=2.188, p=.17$ and $F<1$, respectively]. However, in the accuracy analysis, the main effect of incongruency $[F(1,12)=46.13, p<.001]$ interacted with context, $[F(1$, $12)=14.12, p<.005]$, with more errors on the first relative to last trial in an incongruent run.

This behavioral effect is consistent with both the conflict adaptation theory (less conflict at the end of a run of incongruent trials) and the feature integration theory (Hommel, Proctor, \& Vu, 2004). According to the feature integration account, individuals respond more quickly to an incongruent trial preceded by an incongruent trial because there is either a complete match between stimulus and response features or a complete mismatch. In contrast, an incongruent trial preceded by a congruent trial yields a partial mismatch in terms of either stimulus or the response and so elicits competing reactions.

\section{The Effect of Sustained Incongruency (Trials 2-6)}

In the analyses of task (Stroop vs. Simon) and trial type (incongruent, congruent, neutral) over sustained trials (Trials 2-6 but not 1), participants were less accurate and slower for incongruent trials than congruent or neutral trials. The main effect of trial type (congruent, neutral, and incongruent) was significant for both RTs $[F(1,12)=31.01$, $p<.001]$ and accuracy $[F(1,12)=15.78, p<.001]$ and neither effect interacted with task $(F<1 ; p>.05)$. Individuals were slower $[F(1,12)=16.99]$ and less accurate $[F(1$, $12)=7.19]$ for the Stroop compared to the Simon task (both $p s<.05$ ).

Inspection, and follow-up (nonorthogonal) contrast analyses, indicated a pattern in line with the literature: Incongruent trials were slower than neutral trials in both the Stroop and Simon tasks $[F(1,12)=31.01, p<.001]$, and there was no interaction between trial type and task $(F<1)$.

Incongruent trials were also less accurate than neutral trials in the Stroop task $[F(1,12)=10.20, p<.01]$, but not in the Simon task $(F<1)$, and the difference between the tasks was significant $[F(1,12)=5.25, p<.05]$. In contrast, congruent trials were marginally slower than neutral trials in the Stroop task and showed facilitation only in the Simon task. The interaction between trial type (congruent/ neutral) and task was significant $[F(1,12)=20.33, p=$ .001]. There were no significant differences in congruent and neutral accuracy for either task $(F<1)$. Prior literature (e.g., MacLeod, 1991, pp. 174-175) indicates that facilitation is not an invariable correlate of congruency in the
Stroop task. RTs to congruent trials may not differ from neutral trials (XXXX) or may be longer than to such trials but less than to incongruent trials.

\section{The Onset of Incongruency (i.e., First Trial of a Run)}

The onsets of incongruent and congruent trials were compared following a run of neutral trials versus following a run of mismatching trials (i.e., an incongruent trial following a run of congruent trials and vice versa). The factors in the RT and accuracy ANOVAs were: trial type (congruent/ incongruent), task (Stroop/Simon), and context (neutral/ mismatching). As expected, responses to incongruent trials were slower $[F(1,12)=10.29, p<.01]$ and less accurate $[F(1,12)=63.52, p<.001]$ than congruent trials. Means \pm standard error $=529 \mathrm{msec}$ (19.5) and $70.5 \%$ (3.1\%) for incongruent trials and $493 \mathrm{msec}$ (17.4) and 93.8\% (1.5\%) for congruent trials. These effects did not interact with task or context ( $p>.05$ for all interactions) but there was a main effect of task $[F(1,12)=6.51, p<.05]$, with slower responses in the Stroop task $[M=529$ (22.1)] than in the Simon task $[M=493$ (15.2)].

\section{Local Sequential Modulation of Response}

We checked the correspondence of our data with prior studies showing that individuals adjust their response to incongruent trials (e.g., Kerns, 2006; Kerns et al., 2004). The most direct test of such modulation in our design arises when the type of trial changes following a run of neutral trials such a restriction seems reasonable given the absence of an effect of context in Analysis 3 above. We analyzed mean correct RTs and accuracy of response to the first two congruent or incongruent trials following a run of neutral trials. The ANOVA consisted of the factors task (Stroop/Simon), trial type (congruent/incongruent), and trial position (first/second). If there is modulation of response, the impact of incongruity should be less on the second trial compared to the first, and so there should be a significant interaction between type of trial (congruent or incongruent) and trial position (first or second). In line with expectation, the accuracy rate was higher for the second compared to the first trial in both the congruency [98.7\% (0.4\%) vs. 93.5\% (1.3\%)] and incongruency condition $[91.3 \%$ (0.9\%) vs. $73.4 \%$ (3.3\%)], and this difference was greater for incongruent than congruent trials $[F(1$, $12)=19.92, p=.001]$. There was no corresponding effect on RTs $(F<1)$ and no interaction with task in either the accuracy of RT analysis $[F(1,12)=2.43, p=.15$ for accuracy; and $F<1$ for RT]. Our data are therefore consistent with earlier work and show common effects for the Stroop and Simon tasks.

\section{Response over Sustained Trials}

Responses in choice RT task may also be affected by the sequence of repetition or alternations of response (e.g., 
Smulders et al., 2005; Kirby, 1980). Our study was not designed to examine these effects, and response change occurred unpredictably during the sustained trials. Nonetheless, we felt it desirable to determine if any sequential effects interacted with the type of trial (congruent/incongruent) or task (Stroop/Simon). They did not.

From Figure 4, it is apparent that RT showed an increase over the sustained trials (Positions 2-6) and that this increase was somewhat greater for the Stroop compared to the Simon task. A repeated measures ANOVA of the correct RTs with the factors of task (Stroop/Simon), trial type (congruent/incongruent), and trial position (Positions 2-6) showed significant main effects of trial type $[F(1,12)=$ $73.16, p<.001]$, task $[F(12)=27.36, p<.001]$, and trial position $[F(1,12)=13.74, p<.001]$, in which the latter effect interacted with task $[F(1,12)=3.16, p<.05]$.

RT was significantly slower on incongruent compared to congruent trials $[M=513$ (16.9) vs. 479 (14.7)] and slower on the Stroop compared to the Simon task $[M=524$ (19.5) vs. 468 (13.1)]. The linear increase in RT across trials was more marked for the Stroop compared to the Simon task, as indicated by significant linear component to the interaction variance $[F(1,12)=12.12, p<.05]$. However, critically, this difference between the two tasks did not interact with the type of trial (congruent/incongruent) nor were there any other significant effects (all $p s>.05$ ).

An identical ANOVA on the accuracy data confirmed that responses were more accurate for congruent than incongruent trials $[M=0.964(0.009)$ and $M=0.926(.012)$, respectively; $F(1,12)=24.40, p<.001]$ and for the Simon task compared to the Stroop task $[M=0.961(0.021)$ and $M=0.929$ (0.011), respectively; $F(1,12)=6.43, p<.05]$. There was also a main effect of trial position $[F(4,48)=$ $3.76, p<.05]$ but this did not interact with task $(p>$ $.05)$. The position effect arose from a small reduction in accuracy for the third sustained trial and a larger reduction in accuracy on the fifth sustained trial. There were no other significant main effects or interactions (all $p s>.05$ ).

The increase in RT over the set of trials may reflect the build up of expectations about the likelihood of response repetition or alternation (Smulders et al., 2005; Kirby, 1980) or, more simply, the priming of detectors representing repetitions or alternations (see Cho et al., 2002 for a computational exploration). The increased effect for the Stroop task plausibly indicates the greater difficulty in overcoming an incorrectly primed response.

\section{Acknowledgments}

This work was funded by the Wellcome Trust. We thank Fiona Richardson and Mohamed Seghier for their comments on the manuscript; Norman Price for his help with figure preparation; and our radiographers Amanda Brennan, Janice Glensman, and David Bradbury for their help collecting the data.

Reprint requests should be sent to Prof. Cathy J. Price, Wellcome Trust Centre for Neuroimaging, 12, Queen Square, London WC1N 3BG, UK, or via e-mail: c.price@fil.ion.ucl.ac.uk.

\section{REFERENCES}

Aarts, E., Roelofs, A., \& van Turennout, M. (2008). Anticipatory activity in anterior cingulate cortex can be independent of conflict and error likelihood. Journal of Neuroscience, 28, 4671-4678.

Abel, S., Dressel, K., Bitzer, R., Kümmerer, D., Mader, I., Weiller, C., et al. (2009). The separation of processing stages in a lexical interference fMRI-paradigm. Neuroimage, 44, $1113-1124$

Abutalebi, J., Annoni, J. M., Zimine, I., Pegna, A. J., Seghier, M. L., Lee-Jahnke, H., et al. (2008). Language control and lexical competition in bilinguals: An event-related fMRI study. Cerebral Cortex, 18, 1496-1505.

Abutalebi, J., Brambati, S. M., Annoni, J. M., Moro, A., Cappa, S. F., \& Perani, D. (2007). The neural cost of the auditory perception of language switches: An event-related fMRI study in bilinguals. Journal of Neuroscience, 27, 13762-13769.

Abutalebi, J., \& Green, D. W. (2007). Bilingual speech production: The neurocognition of language representation and control. Journal of Neurolinguistics, 20, 242-275.

Botvinick, M. M., Braver, T. S., Barch, D. M., Carter, C. S., \& Cohen, J. D. (2001). Conflict monitoring and cognitive control. Psychological Review, 108, 624-652.

Bugg, J. M., DeLosh, E. L., Davalos, D. B., \& Davis, H. P. (2007). Age differences in Stroop interference: Contributions of general slowing and task-specific deficits. Neuropsychology, Development, and Cognition: Section B, Aging, Neuropsychology and Cognition, 14, 155-167.

Carter, C. S., \& van Veen, V. (2007). Anterior cingulate cortex and conflict detection: An update of theory and data. Cognitive, Affective \& Behavioral Neuroscience, 7, 367-379.

Chee, M. W. L. (2006). Disassociating language and word meaning in the bilingual brain. Trends in Cognitive Sciences, 10, 527-529.

Cho, R. Y., Nystrom, L. E., Brown, E. T., Jones, A. D., Braver, T. S., Holmes, P. J., et al. (2002). Mechanisms underlying dependencies of performance on stimulus history in a two-alternate forced-choice task. Cognitive, Affective $\mathcal{E}$ Behavioral Neuroscience, 2, 283-299.

Cohen, J. D., Dunbar, K., \& McClelland, J. L. (1990). On the control of automatic processes: A parallel distributed-processing account of the Stroop effect. Psychological Review, 97, 332-361.

Crinion, J., Turner, R., Grogan, A., Hanakawa, T., Noppeney, U., Devlin, T., et al. (2006). Language control in the bilingual brain. Science, 312, 1537-1540.

Delgado, M. R., Miller, M. M., Inati, S., \& Phelps, E. A. (2005). An fMRI study of reward-related probability learning. Neuroimage, 24, 862-873.

Desimone, R., \& Duncan, J. (1995). Neural mechanisms of selective visual attention. Annual Review of Neuroscience, 18, 193-222.

de Zubicaray, G., McMahon, K., Eastburn, M., \& Pringle, A. (2006). Top-down influences on lexical selection during spoken word production: A 4T fMRI investigation of refractory effects in picture naming. Human Brain Mapping, 27, 864-873.

Durston, S., Davidson, M. C., Thomas, K. M., Worden, M. S., Tottenham, N., Martinez, A., et al. (2003). Parametric manipulation of conflict and response competition using rapid mixed-trial event-related fMRI. Neuroimage, 20, $2135-2141$.

Egner, T., Delano, M., \& Hirsch, J. (2007). Separate conflict-specific cognitive control mechanisms in the human brain. Neuroimage, 35, 940-948. 
Egner, T., \& Hirsch, J. (2005a). The neural correlates and functional integration of cognitive control in a Stroop task. Neuroimage, 24, 539-547.

Egner, T., \& Hirsch, J. (2005b). Cognitive control mechanisms resolve conflict through cortical amplification of task-relevant information. Nature Neuroscience, 8, 1784-1790.

Fan, J., Flombaum, J. I., McCandliss, B. D., Thomas, K. M., \& Posner, M. I. (2003). Cognitive and brain consequences of conflict. Neuroimage, 18, 42-57.

Fan, J., Hof, P. R., Guise, K. G., Fossella, J. A., \& Posner, M. I. (2008). The functional integration of the anterior cingulate cortex during conflict processing. Cerebral Cortex, 18, 796-805.

Friederici, A. D. (2006). What's in control of language? Nature Neuroscience, 9, 991-992.

Friston, K. J., Zarahn, E., Josephs, O., Henson, R. N., \& Dale, A. M. (1999). Stochastic designs in event-related fMRI. Neuroimage, 10, 607-619.

Gil Robles, S., Gatignol, P., Capelle, L., Mitchell, M.-C., \& Duffau, H. (2005). The role of dominant striatum in language: A study using intraoperative electrical stimulations. Journal of Neurology, Neurosurgery and Psychiatry, 76, 940-946.

Green, D. W. (1998). Mental control of the bilingual lexico-semantic system. Bilingualism: Language and Cognition, 1, 67-81.

Green, D. W., \& Price, C. J. (2001). Functional imaging in the study of recovery patterns in bilingual aphasia. Bilingualism: Language and Cognition, 4, 191-201.

Harrison, B. J., Shaw, M., Yücel, M., Purcell, R., Brewer, W. J., Strother, S. C., et al. (2005). Functional connectivity during Stroop task performance. Neuroimage, 24, 181-191.

Haupt, S., Axmacher, N., Cohen, M. X., Elger, C. E., \& Fell, J. (2009). Activation of the caudal anterior cingulate cortex due to task-related interference in an auditory Stroop paradigm. Human Brain Mapping, 30, 3043-3056.

Hocking, J., McMahon, K. L., \& de Zubicaray, G. I. (2008). Semantic context and visual feature effects in object naming: An fMRI study using arterial spin labeling. Journal of Cognitive Neuroscience, 21, 1571-1583.

Hoenig, K., \& Scheef, L. (2009). Neural correlates of semantic ambiguity processing during context verification. Neuroimage, 45, 1009-1019.

Hommel, B., Proctor, R. W., \& Vu, K. P. (2004). A feature-integration account of sequential effects in the Simon task. Psychological Research, 68, 1-17.

Kastner, S., \& Ungerleider, L. G. (2000). Mechanisms of visual attention in the human cortex. Annual Review of Neuroscience, 23, 315-341.

Kerns, J. G. (2006). Anterior cingulate and prefrontal cortex activity in an fMRI study of trial-to-trial adjustments on the Simon task. Neuroimage, 33, 399-405.

Kerns, J. G., Cohen, J. D., MacDonald, A. W., Cho, R. Y., Stenger, V. A., \& Carter, C. S. (2004). Anterior cingulate conflict monitoring and adjustments in control. Science, 303, 1023-1026.

Kirby, N. (1980). Sequential effects in choice reaction time. London: Academic Press.

Langenecker, S. A., Nielson, K. A., \& Rao, S. M. (2004). fMRI of healthy older adults during Stroop interference. Neuroimage, 21, 192-200.

Li, C. S., Yan, P., Sinha, R., \& Lee, T. W. (2008). Subcortical processes of motor response inhibition during a stop signal task. Neuroimage, 41, 1352-1363.

Liu, J., Bai, J., \& Zhang, D. (2008). Cognitive control explored by linear modeling behavior and fMRI data during Stroop tasks. Physiological Measurement, 29, 703-710.

Liu, X., Banich, M. T., Jacobson, B. L., \& Tanabe, J. L. (2004). Common and distinct neural substrates of attentional control in an integrated Simon and spatial Stroop task as assessed by event-related fMRI. Neuroimage, 22, 1097-1106.

Lu, C. H., \& Proctor, R. W. (1995). The influence of irrelevant location information on performance: A review of the Simon and spatial Stroop effects. Psychonomic Bulletin E Review, 2, 174-207.

Lungu, O. V., Binenstock, M. M., Pline, M. A., Yeaton, J. R., \& Carey, J. R. (2007). Neural changes in control implementation of a continuous task. Journal of Neuroscience, 27, 3010-3016.

MacLeod, C. M. (1991). Half a century of research on the Stroop effect: An integrative review. Psychological Bulletin, 109, 163-203.

Mason, R. A., \& Just, M. A. (2007). Lexical ambiguity in sentence comprehension. Brain Research, 1146, 115-127.

Mead, L. A., Mayer, A. R., Bobholz, J. A., Woodley, S. J., Cunningham, J. M., Hammeke, T. A., et al. (2002). Neural basis of the Stroop interference task: Response competition or selective attention? Journal of the International Neuropsychological Society, 8, 735-742.

Mechelli, A., Henson, R. N., Price, C. J., \& Friston, K. J. (2003). Comparing event-related and epoch analysis in blocked design fMRI. Neuroimage, 18, 806-810.

Melcher, T., \& Gruber, O. (2006). Oddball and incongruity effects during Stroop task performance: A comparative fMRI study on selective attention. Brain Research, 1121, 136-149.

Melcher, T., \& Gruber, O. (2009). Decomposing interference during Stroop performance into different conflict factors: An event-related fMRI study. Cortex, 45, 189-200.

Monchi, O., Petrides, M., Strafella, A. P., Worsley, K. J., \& Doyon, J. (2006). Functional role of the basal ganglia in the planning and execution of actions. Annals of Neurology, 59, 257-264.

Nieuwenhuis, S., \& Yeung, N. (2005). Neural mechanisms of attention and control: Losing our inhibitions? Nature Neuroscience, 8, 1631-1633.

Orr, J. M., \& Weissman, D. H. (2009). Anterior cingulate cortex makes 2 contributions to minimizing distraction. Cerebral Cortex, 19, 703-711.

Parsons, M. W., Harrington, D. L., \& Rao, S. M. (2005). Distinct neural systems underlie learning visuomotor and spatial representations of motor skills. Human Brain Mapping, 24, 229-247.

Peterson, B. S., Kane, M. J., Alexander, G. M., Lacadie, C., Skudlarski, P., Leung, H. C., et al. (2002). An event-related functional MRI study comparing interference effects in the Simon and Stroop tasks. Cognitive Brain Research, 13, 427-440.

Peterson, B. S., Skudlarski, P., Gatenby, J. C., Zhang, H., Anderson, A. W., \& Gore, J. C. (1999). An fMRI study of Stroop word-color interference: Evidence for cingulate subregions subserving multiple distributed attentional systems. Biological Psychiatry, 45, 1237-1258.

Polk, T. A., Drake, R. M., Jonides, J. J., Smith, M. R., \& Smith, E. E. (2008). Attention enhances the neural processing of relevant features and suppresses the processing of irrelevant features in humans: A functional magnetic resonance imaging study of the Stroop task. Journal of Neuroscience, 28, 13786-13792.

Price, C. J., Green, D. W., \& von Studnitz, R. (1999). A functional imaging study of translation and language switching. Brain, 122, 2221-2235.

Price, C. J., Wise, R. J., \& Frackowiak, R. S. (1996). Demonstrating the implicit processing of visually presented words and pseudowords. Cerebral Cortex, 6, 62-70.

Rahman, R. A., \& Melinger, A. (2008). Enhanced phonological facilitation and traces of concurrent word form activation in speech production: An object-naming study with 
multiple distractors. Quarterly Journal of Experimental Psychology, 61, 1410-1440.

Roberts, K. L., \& Hall, D. A. (2008). Examining a supramodal network for conflict processing: A systematic review and novel functional magnetic resonance imaging data for related visual and auditory Stroop tasks. Journal of Cognitive Neuroscience, 20, 1063-1078.

Rodd, J. M., Davis, M. H., \& Johnsrude, I. S. (2005). The neural mechanisms of speech comprehension: fMRI studies of semantic ambiguity. Cerebral Cortex, 15, 1261-1269.

Rodriguez-Fornells, A., Rotte, M., Heinze, H. J., Nösselt, T., \& Münte, T. F. (2002). Brain potential and functional MRI evidence for how to handle two languages with one brain. Nature, 415, 1026-1029.

Rodriguez-Fornells, A., van der Lugt, A., Rotte, M., Britti, B., Heinze, H. J., \& Münte, T. F. (2005). Second language interferes with word production in fluent bilinguals: Brain potential and functional imaging evidence. Journal of Cognitive Neuroscience, 17, 422-433.

Shadmehr, R., \& Holcomb, H. H. (1999). Inhibitory control of competing motor memories. Experimental Brain Research, 126, 235-251.

Sharot, T., De Martino, B., \& Dolan, R. J. (2009). How choice reveals and shapes expected hedonic outcome. Journal of Neuroscience, 29, 3760-3765.

Shohamy, D., Myers, C. E., Kalanithi, J., \& Gluck, M. A. (2008). Basal ganglia and dopamine contributions to probabilistic category learning. Neuroscience and Biobehavioral Reviews, 32, 219-236.

Shohamy, D., Myers, C. E., Onlaor, S., \& Gluck, M. A. (2004). Role of the basal ganglia in category learning: How do patients with Parkinson's disease learn? Behavioral Neuroscience, 118, 676-686.

Simon, J. R. (1969). Reactions toward source of stimulation. Journal of Experimental Psychology, 81, 174-176.

Smulders, S. F. A., Notebaert, W., Meijer, M., Crone, E. A., van der Molen, M. W., \& Soetens, E. (2005). Sequential effects on speeded information processing: A developmental study. Journal of Experimental Child Psychology, 90, 208-234.
Spalek, K., \& Thompson-Schill, S. L. (2008). Task-dependent semantic interference in language production: An fMRI study. Brain and Language, 107, 220-228.

Stroop, J. R. (1935). Studies of interference in serial verbal reactions. Journal of Experimental Psychology, 18, 643-662.

Sumner, P., Nachev, P., Morris, P., Peters, A. M., Jackson, S. R., Kennard, C., et al. (2007). Human medial frontal cortex mediates unconscious inhibition of voluntary action. Neuron, 54, 697-711.

Tricomi, E., Delgado, M. R., McCandliss, B. D., McClelland, J. L., \& Fiez, J. A. (2006). Performance feedback drives caudate activation in a phonological learning task. Journal of Cognitive Neuroscience, 18, 1029-1043.

Tricomi, E., \& Fiez, J. A. (2008). Feedback signals in the caudate reflect goal achievement on a declarative memory task. Neuroimage, 41, 1154-1167.

van Heuven, W. J. B., Schriefers, H., Dijkstra, T., \& Hagoort, P. (2008). Language conflict in the bilingual brain. Cerebral Cortex, 18, 2706-2718.

Van Orden, G. C. (1987). A ROWS is a ROSE: Spelling, sound, and reading. Memory \& Cognition, 15, 181-198.

van Veen, V., \& Carter, C. S. (2005). Separating semantic conflict and response conflict in the Stroop task: A functional MRI study. Neuroimage, 27, 497-504.

Veltman, D., Mechelli, A., Friston, K. J., \& Price, C. J. (2002). The importance of distributed sampling in blocked functional magnetic resonance imaging designs. Neuroimage, 17, 1203-1206.

Vink, M., Kahn, R. S., Raemaekers, M., van den Heuvel, M., Boersma, M., \& Ramsey, N. F. (2005). Function of striatum beyond inhibition and execution of motor responses. Human Brain Mapping, 25, 336-344.

Wuhr, P., \& Ansorge, U. (2005). Exploring trial-by-trial modulations of the Simon effect. Quarterly Journal of Experimental Psychology: Section A, Human Experimental Psychology, 58, 705-731.

Zempleni, M. Z., Renken, R., Hoeks, J. C., Hoogduin, J. M., \& Stowe, L. A. (2007). Semantic ambiguity processing in sentence context: Evidence from event-related fMRI. Neuroimage, 34, 1270-1279. 